\title{
COMMENT
}

\section{On Bäcklund transformations and identities in bilinear form}

\author{
G Post
}

Faculty of Applied Mathematios, University of Twente, PO Box 217, $7500 \mathrm{AE}$ Enschede, The Netherlands

Received 6 July 1990

\begin{abstract}
For bilinear equations of the form $P(D) f \cdot f=0$ we find all possibilities for rewriting $g^{2} P(D) f \cdot f-f^{2} P(D) g \cdot g=0$ in the form $Q(D) f \cdot g=0$. This is the first step in finding a Bäcklund transformation.
\end{abstract}

\section{Introduction}

In this comment we study Bäcklund transformations in bilinear form. This technique was introduced by Hirota [1,2]. Let us start with a brief sketch of the method, and formulate the questions that we want to answer. We suppose that we are given an equation in bilinear form

$$
P(D) f \cdot f=0
$$

Here $P$ is a polynomial in, say, $n$ variables, i.e.

$$
P\left(D_{1}, \ldots, D_{n}\right)=\sum c_{\alpha} D^{\alpha}
$$

where $\alpha=\left(\alpha_{1}, \ldots, \alpha_{n}\right)$ and $D^{\alpha}=D_{n}^{\alpha_{n}} \ldots D_{1}^{\alpha_{1}}$, where

$$
\begin{aligned}
D_{n}^{\alpha_{n}} \cdots D_{1}^{\alpha_{1}} f & \cdot g=\frac{\partial^{\alpha_{n}}}{\partial y_{n}^{\alpha_{n}}} \cdots \frac{\partial^{\alpha_{1}}}{\partial y_{1}^{\alpha_{1}}} f\left(x_{1}+y_{1}, \ldots, x_{n}+y_{n}\right) \\
& \times\left. g\left(x_{1}-y_{1}, \ldots, x_{n}-y_{n}\right)\right|_{y_{1}=\cdots=y_{n}=0}
\end{aligned}
$$

To find a Bäcklund transformation, we apply the following trick: consider the equation

$$
g^{2} P(D) f \cdot f-f^{2} P(D) g \cdot g=0 .
$$

Then we note that for a solution $f, g$ to (2), the following holds:

$$
f \text { is a solution of }(1) \Leftrightarrow g \text { is a solution of (1). }
$$

Suppose that we could rewrite (2) in the following form:

$$
Q(D)\left[Q_{1}(D)(f \cdot g) \cdot Q_{2}(D)(f \cdot g)\right]=0
$$


Then by suitably splitting $Q$ we derive two equations:

$$
\begin{aligned}
& P_{1}(D) f \cdot g=0 \\
& P_{2}(D) f \cdot g=0 .
\end{aligned}
$$

The system (4) can be called a Bäcklund transformation for $P(D) f \cdot f=0$. Namely, suppose $g$ is given; then a solution $f$ to equation (4) will also satisfy $P(D) f \cdot f=0$.

The process of sensibly splitting $Q$ is strongly equation-dependent; it seems unclear in general how to perform it. Two other general questions remain:

1. Is there for any $P$ a solution $Q$ ?

2. Is this solution unique? If not, can one find all possibilities?

In this comment we answer these questions. The answer to the first is yes; the proof is already essentially in Hirota $[1,2]$. This solution is not at all unique. We give all possibilities in terms of a generating identity. The proof that these are all the possibilities is the most difficult part, and not fully included here. For complete proofs, see [3].

\section{Algebraic background; partial solution}

Let $J$ denote the space of multi-indices $\left(i_{1}, \ldots, i_{n}\right)$. In practice we will encounter $f^{(i)}$ and $g^{(j)}(i, j \in J)$, which will substitute for the partial derivatives. We will deal with the polynomial algebra $\mathcal{A}=\mathbb{R}\left[f^{(i)}, g^{(j)}\right],(i, j \in J)$. In this algebra, we have derivations $\partial_{r}(r=1 \cdots n)$ which act in the obvious way. In particular

$$
\partial_{r}\left(f^{(i)}\right)=f^{(i+1 r)} \text { and } \partial_{r}\left(g^{(j)}\right)=g^{\left(j+1_{r}\right)}
$$

where $1_{r}=(0, \ldots, 0,1,0, \ldots, 0)$, the $r$ th basis vector.

Corresponding to $\partial_{r}$, we introduce the linear map $\mathcal{D}_{r}$ (the Hirota derivative) as a $\operatorname{map} \mathcal{A} \otimes \mathcal{A} \rightarrow \mathcal{A} \otimes \mathcal{A}$, by

$$
\mathcal{D}_{r}(a \otimes b)=\partial_{r}(a) \otimes b-a \otimes \partial_{r}(b) \quad(r=1, \ldots, n)
$$

The derivative $\partial_{r}$ is also extended to $\mathcal{A} \otimes \mathcal{A}$ by

$$
\partial_{r}(a \otimes b)=\partial_{r}(a) \otimes b+a \otimes \partial_{r}(b) .
$$

Obviously, we can define a Hirota derivative corresponding to any derivation of $\mathcal{A}$.

Lemma 1. Let $\mathcal{D}=\mathcal{D}_{r}$ and $\partial=\partial_{r}$ and $\mathcal{A}$ be as above. Then

$$
\exp (\epsilon \mathcal{D})(a \otimes b)=\exp (\epsilon \partial) a \otimes \exp (-\epsilon \partial) b \text {. }
$$

The equality is meant as formal power series, and follows by computing homogeneous terms with reference to $\epsilon^{i}, i=0,1,2, \ldots$.

Note that our definition of $\mathcal{D}_{r}$ differs from the usual one in the following sense: the image is again in $\mathcal{A} \otimes \mathcal{A}$ and not in $\mathcal{A}$. This is a major difference, as we will see shortly. To get the usual Hirota derivatives $D_{r}$ we have to project the image of $\mathcal{D}_{r}$ to $\mathcal{A}:$ let $\pi: \mathcal{A} \otimes \mathcal{A} \rightarrow \mathcal{A}, \pi\left(\sum a_{i} \otimes b_{i}\right)=\sum a_{i} b_{i}$, denote this projection. Then

$$
D_{r}(a \otimes b):=\pi \mathcal{D}_{r}(a \oslash b)
$$

and more generally

$$
D_{r}^{k}(a \otimes b):=\pi \mathcal{D}_{r}^{k}(a \otimes b), k=1,2, \ldots
$$

The exchange formula is central in this comment. 
Theorem 1 (cf Hirota). Let $\partial_{\alpha}=\sum \alpha_{i} \partial_{i}, \partial_{\beta}=\sum \beta_{i} \partial_{i}$ and $\partial_{\gamma}=\sum \gamma_{i} \partial_{i}$ and let $D_{\alpha}$ etc. be the corresponding (projected) Hirota derivatives. Then we have for all $a, b, c, d \in \mathcal{A}$

$$
\begin{aligned}
& \exp \left(D_{\alpha}\right)\left(\exp \left(D_{\beta}\right)(a \otimes b) \otimes \exp \left(D_{\gamma}\right)(c \otimes d)\right) \\
&=\exp \left(\frac{1}{2}\left(D_{\beta}-D_{\gamma}\right)\right)\left(\exp \left(\frac{1}{2}\left(D_{\beta}+D_{\gamma}\right)+D_{\alpha}\right)(a \otimes d)\right. \\
&\left.\otimes \exp \left(\frac{1}{2}\left(D_{\beta}+D_{\gamma}\right)-D_{\alpha}\right)(c \otimes b)\right)
\end{aligned}
$$

Proof. The proof is based on lemma 1: expressing all the $\mathcal{D}$ in their corresponding $\partial$ and projecting on $\mathcal{A}$ gives the result immediately.

Note that this identity solves our first question by taking $a=b=f$ and $c=d=g$. Using the fact that $\exp \left(\sum \epsilon_{i} \mathcal{D}_{i}\right)=\prod \exp \left(\epsilon_{i} \mathcal{D}_{i}\right)$, we see that for three multi-indices $k, l, m \in J$, the coefficient of $\alpha^{k} \beta^{l} \gamma^{m}$ in (5) expresses

$$
D^{k}\left(D^{l}(f \cdot f) \cdot D^{m}(g \cdot g)\right)
$$

in terms of

$$
D \cdot(D \cdot(f \cdot g) \cdot D \cdot(g \cdot f))
$$

Moreover we note that such an expression is not unique. If we take $a=c=f$ and $b=d=g$, then again comparing the coefficients of $\alpha^{k} \beta^{l} \gamma^{m}$ in (5), we see that $D^{k}\left(D^{l}(f \cdot g) \cdot D^{m}(f \cdot g)\right)$ can be re-expressed. These observations solve a part of our problem; however, they do not solve the most difficult part. It is important to find all possibilities, hence all identities of the form

$$
D^{k}\left(D^{l}(f \cdot g) \cdot D^{m}(f \cdot g)\right)=\sum D \cdot(D \cdot(f \cdot g) \cdot D \cdot(f \cdot g)) .
$$

This problem will be solved in the next section. The answer is slightly surprising: equation (5) already contains all non-trivial identities!

\section{Finding all identities}

To study the identities of the form (6) more closely, we introduce two subspaces of $\mathcal{A} \otimes \mathcal{A}$. The first one, denoted by $\mathcal{B}$, is the linear space spanned by the elements $\left\{f^{(i)} \otimes g^{(j)}\right\}, \quad(i, j \in J)$. Clearly these elements form a basis. Note that $\left.\pi\right|_{\mathcal{B}}$ is injective. This allows us to view $\mathcal{B}$ as a subspace of $\mathcal{A}$. We introduce

$$
e_{\alpha}^{(\beta)}:=\partial^{\beta} \mathcal{D}^{\alpha}(f \otimes g)
$$

which are again elements of $\mathcal{B}$. For these elements one can prove the following lemma.

Lemma 2. $\left\{e_{\alpha}^{(\beta)}\right\}$ is a basis for $\mathcal{B}$.

Proof. The proof (by induction on the number of independent variables $n$ ) is based on the observation that $\mathcal{D}^{\alpha}(f \otimes g) \notin \sum_{r=1}^{n} \operatorname{Im}\left(\partial_{r}\right)$. 
Identifying $\mathcal{B}$ with a subspace of $\mathcal{A}$, we can define $\mathcal{D}_{r}$ (and $\left.\partial_{r}\right): \mathcal{B} \otimes \mathcal{B} \rightarrow \mathcal{B} \otimes \mathcal{B}$. In $\mathcal{B} \otimes \mathcal{B}$ we define the subspace $\mathcal{C}$, spanned by the elements

$$
\mathcal{D}^{k}\left(\mathcal{D}^{l}(f \cdot g) \otimes \mathcal{D}^{m}(f \cdot g)\right) \quad(k, l, m \in J) .
$$

Thanks to lemma 2, these elements are linearly independent, i.e. they form a basis for $\mathcal{C}$. This shows that finding identities of the form (6) is equivalent to finding $\operatorname{ker}(\pi) \mid \mathcal{C}$. Before turning to $\operatorname{ker}(\pi)$ we mention:

Lemma 3. Let $\mathcal{B}$ and $\mathcal{C}$ be as above. Then $\mathcal{B} \otimes \mathcal{B}=\mathcal{C} \oplus \operatorname{Im}(\partial)$, where $\operatorname{Im}(\partial):=$ $\sum_{r=1}^{n} \operatorname{Im}\left(\partial_{r}\right)$.

Expressed in normal derivatives, $\left.\operatorname{ker}(\pi)\right|_{\mathcal{B} \otimes} \mathcal{B}$ is easily described.

Lemma 4. $\left.\quad \operatorname{ker}(\pi)\right|_{\mathcal{B} \otimes \mathcal{B}}$ is generated by

1.

$$
\begin{array}{ll}
\text { 1. } & f^{(i)} g^{(j)} \otimes f^{(k)} g^{(l)}-f^{(k)} g^{(l)} \otimes f^{(i)} g^{(j)} \\
\text { 2. } & f^{(i)} g^{(j)} \otimes f^{(k)} g^{(l)}-f^{(i)} g^{(l)} \otimes f^{(k)} g^{(j)}
\end{array}
$$

The elements mentioned under 1 give rise to trivial identities, namely the identities

$$
D^{k}\left(D^{l}(f \cdot g) \otimes D^{m}(f \cdot g)\right)=(-1)^{|k|} D^{k}\left(D^{m}(f \cdot g) \otimes D^{l}(f \cdot g)\right) .
$$

To analyse the elements under 2 , we introduce $d_{r}$ and $\bar{d}_{r}: \mathcal{B} \rightarrow \mathcal{B}$, defined by

$$
d_{r}\left(f^{(i)} g^{(j)}\right)=f^{\left(i+1_{r}\right)} g^{(j)}, \bar{d}_{r}\left(f^{(i)} g^{(j)}\right)=f^{(i)} g^{(j+1 r)}
$$

The elements $f^{(i)} g^{(j)} \otimes f^{(k)} g^{(l)}-f^{(i)} g^{(l)} \otimes f^{(k)} g^{(j)}$ are precisely the homogeneous terms in

$$
\begin{aligned}
\exp \left(\sum _ { r = 1 } ^ { n } \left(\alpha_{r} d_{r}\right.\right. & \left.\left.+\beta_{r} \bar{d}_{r}\right)\right) f \cdot g \otimes \exp \left(\sum_{r=1}^{n}\left(\gamma_{r} d_{r}+\delta_{r} \bar{d}_{r}\right)\right) f \cdot g \\
& -\exp \left(\sum_{r=1}^{n}\left(\alpha_{r} d_{r}+\delta_{r} \bar{d}_{r}\right)\right) f \cdot g \otimes \exp \left(\sum_{r=1}^{n}\left(\gamma_{r} d_{r}+\beta_{r} \bar{d}_{r}\right)\right) f \cdot g
\end{aligned}
$$

Using $\partial_{r}=d_{r}+\bar{d}_{r}$ and $\mathcal{D}_{r}=d_{r}-\bar{d}_{r}$, we can rewrite $(7)$ in terms of $\partial_{r}$ and $\mathcal{D}_{r}:(7)$ turns into

$$
\begin{aligned}
& \exp \left(\frac{1}{4} \sum\left(\alpha_{r}+\beta_{r}+\gamma_{r}+\delta_{r}\right) \partial_{r}\right)\left\{\exp \left(\frac{1}{4} \sum\left(\alpha_{r}+\beta_{r}-\gamma_{r}-\delta_{r}\right) \mathcal{D}_{r}\right)\right. \\
&\left(\exp \left(\frac{1}{2} \sum\left(\alpha_{r}-\beta_{r}\right) \mathcal{D}_{r}\right)(f \cdot g) \otimes \exp \left(\frac{1}{2} \sum\left(\gamma_{r}-\delta_{r}\right) \mathcal{D}_{r}\right)(f \cdot g)\right) \\
&-\exp \left(\frac{1}{4} \sum\left(\alpha_{r}+\delta_{r}-\gamma_{r}-\beta_{r}\right) \mathcal{D}_{r}\right)\left(\exp \left(\frac{1}{2} \sum\left(\alpha_{r}-\delta_{r}\right) \mathcal{D}_{r}\right)(f \cdot g)\right. \\
&\left.\left.\otimes \exp \left(\frac{1}{2} \sum\left(\gamma_{r}-\beta_{r}\right) \mathcal{D}_{r}\right)(f \cdot g)\right)\right\} .
\end{aligned}
$$


Changing variables

$$
\begin{array}{ll}
\bar{\delta}_{r}=\frac{1}{4}\left(\alpha_{r}+\beta_{r}+\gamma_{r}+\delta_{r}\right) & \bar{\alpha}_{r}=\frac{1}{4}\left(\alpha_{r}+\beta_{r}-\gamma_{r}-\delta_{r}\right) \\
\bar{\beta}_{r}=\frac{1}{2}\left(\alpha_{r}-\beta_{r}\right) & \bar{\gamma}_{r}=\frac{1}{2}\left(\gamma_{r}-\delta_{r}\right)
\end{array}
$$

expression (8) transforms into

$$
\begin{aligned}
\exp \left(\sum \bar{\delta}_{r} \partial_{r}\right) & \left\{\exp \left(\sum \bar{\alpha}_{r} \mathcal{D}_{r}\right)\left(\exp \left(\sum \bar{\beta}_{r} \mathcal{D}_{r}\right)(f \cdot g) \otimes \exp \left(\sum \bar{\gamma}_{r} \mathcal{D}_{r}\right)(f \cdot g)\right)\right. \\
& -\exp \left(\frac{1}{2} \sum\left(\bar{\beta}_{r}-\bar{\gamma}_{r}\right) \mathcal{D}_{r}\right)\left(\exp \left(\frac{1}{2} \sum\left(2 \bar{\alpha}_{r}+\bar{\beta}_{r}+\bar{\gamma}_{r}\right) \mathcal{D}_{r}\right)(f \cdot g)\right. \\
& \left.\left.\otimes \exp \left(\frac{1}{2} \sum\left(-2 \bar{\alpha}_{r}+\bar{\beta}_{r}+\bar{\gamma}_{r}\right) \mathcal{D}_{r}\right)(f \cdot g)\right)\right\}
\end{aligned}
$$

Since (9) is an invertible transformation, the homogeneous terms in (10) span the same space as in $(7)$. For $\left.\operatorname{ker}(\pi)\right|_{\mathcal{C}}$ we only need to consider the coefficients in which $\bar{\delta}$ does not appear (see lemma 3 ). Hence we are left with the expression between braces, which is identical to theorem 1 for $a=c=f$ and $b=d=g$. So these (and the trivial ones) are all identities.

I would like to thank Professors Conte and Martini for drawing my attention to this problem.

\section{References}

[1] Hirota $\mathrm{R} 1974 \mathrm{~A}$ new form of Bäcklund transformations and its relation to the inverse scattering problem Prog. Theor. Phys. 52 1498-512

[2] Hirota R 1980 Direct methods in soliton theory Solitons ed R K Bullough and P J Caudrey (Berlin: Springer)

[3] Post G 1990 Bäcklund transformations and identities in bilinear form Memorandum 856 University of Twente, The Netherlands 